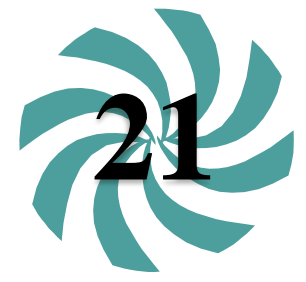

Tecnociencia, Vol. 23, $\mathrm{N}^{\circ} 1: 385-403$

Enero-Junio 2021

\title{
DIVERSIDAD DE MARIPOSAS (LEPIDOPTERA: PAPILIONOIDEA Y HESPERIOIDEA) EN LA MESETA DE CHORCHA, PROVINCIA DE CHIRIQUí, PANAMÁ.
}

\author{
${ }^{1}$ Gabriel Araúz (D), ${ }^{2}$ Carlos Iglesias $\left(\mathrm{D},{ }^{3}\right.$ Alonso Santos M. (iD $\mathrm{R}^{\circ}$, \\ ${ }^{4}$ Yostin Añino $i \mathrm{R} \mathrm{R}^{0}$. \\ ${ }^{1,2}$ Universidad Autónoma de Chiriquí, Museo de Historia Natural, Facultad de \\ Ciencias Naturales y Exactas, e-mail: g_arauz10@hotmail.com; ${ }^{3,4}$ Universidad de \\ Panamá, Museo de Invertebrados G.B. Fairchild, Facultad de Ciencias Naturales, \\ Exactas y Tecnología. E-mails: Alonso Santos Murgas: alonso.santos@up.ac.pa y \\ santosmurgasa@gmail.com; ${ }^{4}$ Programa de Maestría en Gestión Ambiental y \\ Sostenibilidad, Universidad del Istmo, Panamá.
}

\section{RESUMEN}

Se determinó la diversidad de mariposas en el trayecto principal hacia la meseta de Chorcha, ubicada en los distritos de David, Gualaca y San Lorenzo, provincia de Chiriquí, Panamá. Se realizaron 24 giras de colecta durante tres meses de la época lluviosa de 2017 y tres meses de la época seca de 2018. Para esto se utilizó el método de captura directa con la red entomológica y 16 trampas Van Someren-Rydon con cebos de fruta descompuesta, fruta fresca, pescado en descomposición y heces de ganado. Se recolectaron 907 individuos, pertenecientes a 112 especies, 6 familias y 21 subfamilias. El método más efectivo para la captura de mariposas fue la red entomológica, con la cual se registraron 757 individuos, en comparación con los 150 individuos colectados con las trampas Van Someren-Rydon. Los valores más altos de abundancia y riqueza de especies se registraron en la época lluviosa con 554 individuos y 168 especies respectivamente. Según los resultados, la meseta de Chorcha presenta una elevada abundancia y riqueza de mariposas, a pesar de que la zona ha sido frecuentemente intervenida por el hombre para la ganadería y la ubicación de las torres de alta tensión eléctrica.

\section{PALABRAS CLAVES}

Mariposas, trampas Van Someren - Rydon, cebos, red entomológica. 


\title{
DIVERSITY OF BUTTERFLIES (LEPIDOPTERA: PAPILIONOIDEA AND HESPERIOIDEA) IN THE PLATEAU OF CHORCHA, PROVINCE OF CHIRIQUÍ, PANAMA.
}

\begin{abstract}
We determined the diversity of butterflies on the main route to Chorcha, located in David's districts, Gualaca and San Lorenzo, Chiriquí, Panama. We made 24 visits to the field, during three months of the rainy season of 2017 and three months of the dry season of 2018. We used the direct capture method with the entomological net and 16 Van Someren-Rydon traps with decomposed fruit, fresh fruit, decomposing fish, and livestock feces as baits. 907 individuals were collected, belonging to 112 species, 6 families, and 21 subfamilies. The most effective method for capturing butterflies was the entomological net, with which 757 individuals were registered, compared to the 150 individuals collected with the Van Someren-Rydon traps. The highest abundance and richness of species were identified in the rainy season with 554 individuals and 168 species, respectively. According to the results, Chorcha has a high abundance and richness of butterflies, even though the area has been frequently intervened by man for livestock and the location of high voltage towers.
\end{abstract}

\section{KEYWORDS}

Butterflies, Van Someren - Rydon traps, baits, entomological net.

\section{INTRODUCCIÓN}

El orden Lepidoptera constituye un numeroso grupo de insectos que cuenta con más 150000 especies descritas a nivel mundial (GarcíaBarros et al., 2015). Urretabizkaya (2010), señala que los miembros de este grupo incluyen mariposas y polillas o mariposas nocturnas. En Panamá, la diversidad de lepidópteros abarca más 16000 especies (Aiello, 2001), de las cuales 550 pertenecen al grupo de mariposas (DeVries, 1987).

Las mariposas han sido ampliamente utilizadas para estudios de biodiversidad, esto se debe a que participan en numerosas interacciones ecológicas, siendo principalmente la polinización de las plantas, el desempeño de un papel importante en la cadena alimenticia y el correcto funcionamiento de los ecosistemas (Ollerton, 1999).

En Panamá se han realizado varios estudios sobre mariposas (Huntington, 1932; Silberglied et al., 1980; Mallet, 1986; Aiello, 1992, 2001; Santos \& Cambra, 2003; Santos, 2012; Basset et al., 2015; Padilla

et al., 2020). Los estudios realizados hasta ahora en la meseta de Tecnociencia, Vol. 23, $N^{\circ} 1$ 
Chorcha son escasos, la única investigación sobre mariposas en esta región es la realizada por Acosta (2007). El autor hizo un inventario sobre la diversidad y abundancia de mariposas y polillas de hábitos diurnos durante los años 2005-2006. El presente estudio pretende dar a conocer la diversidad de mariposas Papilionoidea y Hesperoidea en el trayecto principal hacia la meseta de Chorcha durante tres meses de la época lluviosa de 2017 y tres, de la época seca de 2018.

\section{MATERIALES Y MÉTODOS}

Área de estudio

La investigación fue realizada en el trayecto principal de $4 \mathrm{~km}$ que conduce hacia la meseta de Chorcha, ubicada entre los distritos de David, Gualaca y San Lorenzo, entre las coordenadas geográficas $8^{\circ}$ $24.5^{\prime}-8^{\circ} 27^{\prime} \quad \mathrm{N}$ y $82^{\circ} 11.5^{\prime}-82^{\circ} 14^{\prime} \mathrm{O}$ (Fig. 1). La meseta de Chorcha presenta una extensión de $11 \mathrm{~km}^{2}$ (Castro, 1986) y una elevación entre los 400 y 494 msnm. (Consejo Municipal de David, 2017). Según las zonas de vida de Holdridge (1966), presenta un bosque húmedo tropical y áreas de sabana arbustiva en la superficie con formación boscosa moderadamente densa sobre las laderas (Castro, 1986). Además, presenta una temperatura anual de $27.8{ }^{\circ} \mathrm{C}$ y una precipitación promedio anual de 173.03 mm (ETESA, 2020).

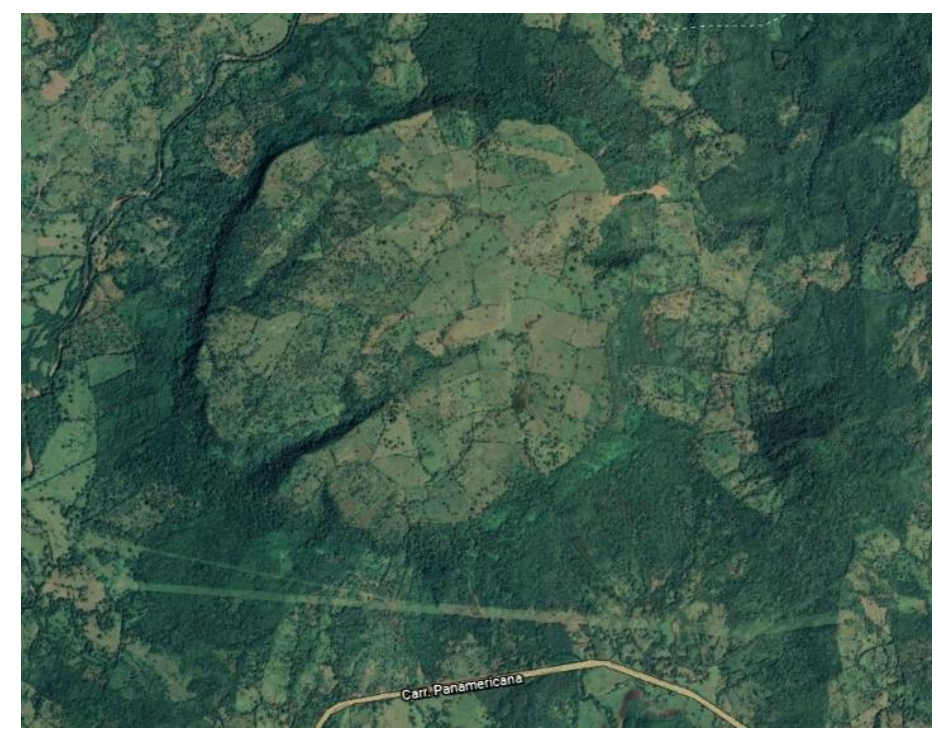

Fig. 1. Vista satelital de la meseta de Chorcha. Fuente: Google Earth. 


\section{Muestreos}

Los muestreos fueron realizados durante tres meses de la época lluviosa de 2017 (de agosto a octubre) y tres meses de la época seca de 2018 (de enero a marzo). Con una frecuencia de una vez por semana, para un total de 24 días de muestreo; participaron tres personas, con un esfuerzo por día de muestreo de 9 horas/hombre y un total de 108 horas/hombre por cada época de muestreo.

El muestreo con la red entomológica fue realizado en el trayecto principal entre las 07:00 h y 16:00 h., para la colocación de las trampas Van Someren-Rydon (VSR), se establecieron cuatro estaciones en áreas paralelas al trayecto principal. Se utilizaron los métodos descritos por Villareal et al. (2004), utilizando 16 trampas VSR, ubicadas a una altura de 1,5 metros del suelo y 50 metros entre sí, en un horario entre las 07:30 h y 15:30 h. Cada una de las trampas de cada estación fueron cebadas con fruta descompuesta (guineo fermentado con levadura y azúcar), fruta fresca (piña, papaya y naranja), pescado descompuesto y heces de ganado.

Las mariposas capturadas, tanto con la red entomológica como con las trampas fueron procesadas en el laboratorio de zoología de la Universidad Autónoma de Chiriquí (UNACHI). Para la identificación de los especímenes se utilizaron claves taxonómicas de Andrade-C (1990) y Rodríguez (1996), además de las guías visuales de DeVries (1987), DeVries (1997), Warren et. al (2016). Los especímenes colectados permanecen en el Museo de Historia Natural (MUHISNAT) de la UNACHI.

Análisis de los resultados:

Se realizaron análisis de diversidad Alpha, destacando el índice de Shannon-Wever y el índice de Simpson, los datos fueron analizados mediante Past 3 y fueron tabulados usando el programa Microsoft Excel 2013.

\section{RESULTADOS}

Se recolectaron 907 individuos pertenecientes a 112 especies, 77 géneros, 21 subfamilias y seis familias. La mayor abundancia de mariposas se registró en la época lluviosa de 2017 y la menor abundancia se registró en la época seca de 2018. El mes que presentó 
la mayor riqueza de especies fue agosto con 61 especies, mientras que el mes con la menor riqueza fue enero con 37 especies (Cuadro 1).

Cuadro 1. Abundancia y riqueza de mariposas en el trayecto principal hacia la meseta de Chorcha, durante la época lluviosa de 2017 y la época seca de 2018.

\begin{tabular}{|c|c|c|c|}
\hline Año & Mes & Abundancia & Riqueza \\
\hline \multirow{3}{*}{ 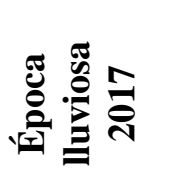 } & Agosto & 187 & 61 \\
\hline & Septiembre & 190 & 60 \\
\hline & Octubre & 177 & 47 \\
\hline \multirow{3}{*}{ 惡 } & Enero & 110 & 37 \\
\hline & Febrero & 96 & 40 \\
\hline & Marzo & 147 & 52 \\
\hline
\end{tabular}

Se determinó el índice de diversidad de Shannon-Weaver $\left(\mathrm{H}^{\prime}\right)$ por mes de muestreo, siendo marzo el mes más diverso $\left(\mathrm{H}^{\prime}=3.59\right)$ y octubre menos diverso $\left(\mathrm{H}^{\prime}=3.17\right)$. El índice de Simpson (1-D) muestra la misma tendencia para marzo $(1-\mathrm{D}=0.96)$ y octubre $(1-\mathrm{D}=0.92)$.

Las mariposas más frecuentes por época y día de muestreo fueron: Morpho helenor (especie más frecuente con 23 registros), Heliconius melpomene, Heraclides thoas, Anartia fatima, Heliconius hecale y Pierella luna, cada una con 22 registros (Fig. 7, 8, 9).

Los valores más altos de abundancia y riqueza de especies se registraron en la época lluviosa con 554 individuos y 168 especies respectivamente; en tanto, durante la época seca se registraron 353 individuos y 129 especies. Cabe destacar que se tomaron en cuenta las variables ambientales de precipitación y temperatura (Fig. 2). 


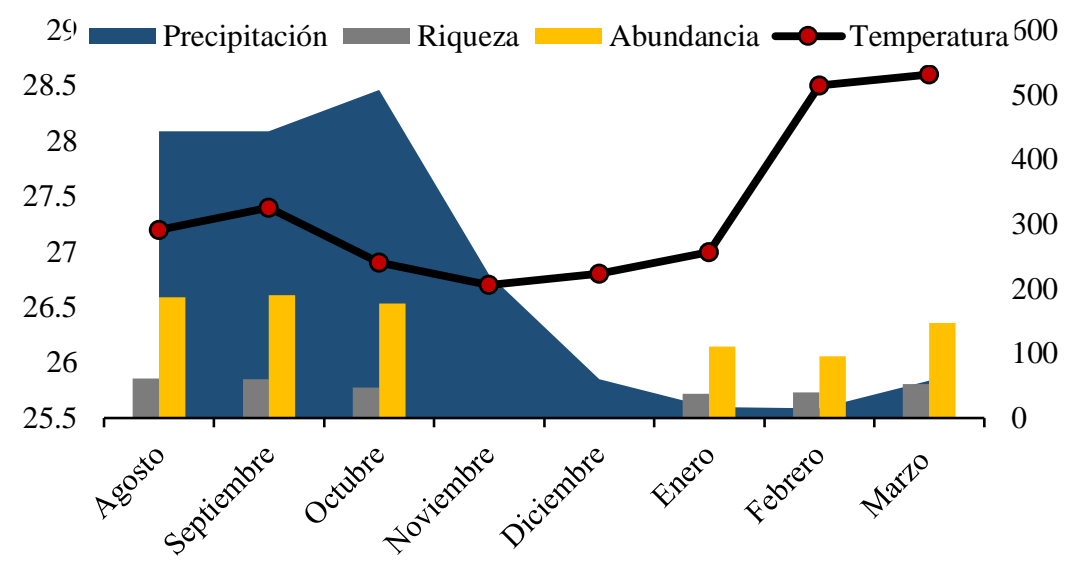

Fig. 2. Abundancia, riqueza y variables ambientales (precipitación y temperatura) por época de muestreo

Se capturaron 757 individuos con la red entomológica y 150 con las trampas VSR, siendo el cebo de la fruta fermentada la más efectiva con 70 individuos distribuidos en 13 especies, el cebo de pescado descompuesto presentó la menor efectividad con 15 individuos y 10 especies (Fig. 3).

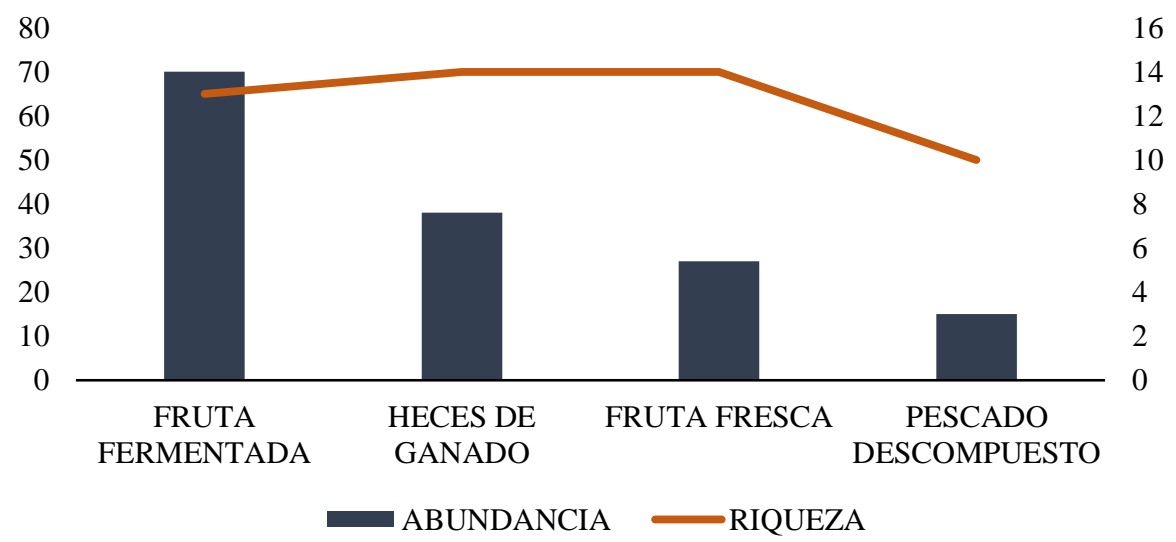

Fig. 3. Abundancia y riqueza de especies según el tipo de cebo de las trampas Van Someren-Rydon

La red entomológica presentó la mayor abundancia y riqueza de las familias de mariposas colectadas en comparación con las trampas (Fig. 4). De los 150 individuos colectados con las trampas, la familia más abundante fue Nymphalidae con 116 individuos y 27 especies, mientras 
que las familias Papilionidae y Lycaenidae fueron las menos abundantes con un individuo y especie respectivamente (Fig. 5).

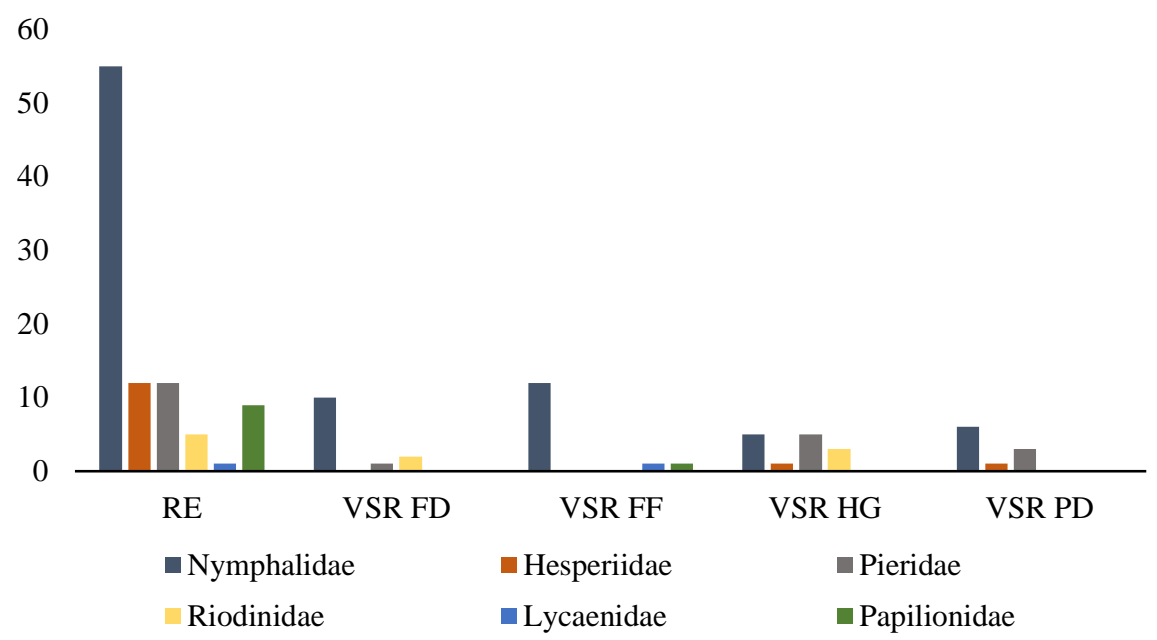

Fig. 4. Riqueza de especies por familia, según el método de colecta. (RE: red entomológica, VSR: trampa Van Someren Rydon, FD: fruta descompuesta, FF: fruta fresca, HG: heces de ganado, PD: pescado descompuesto).

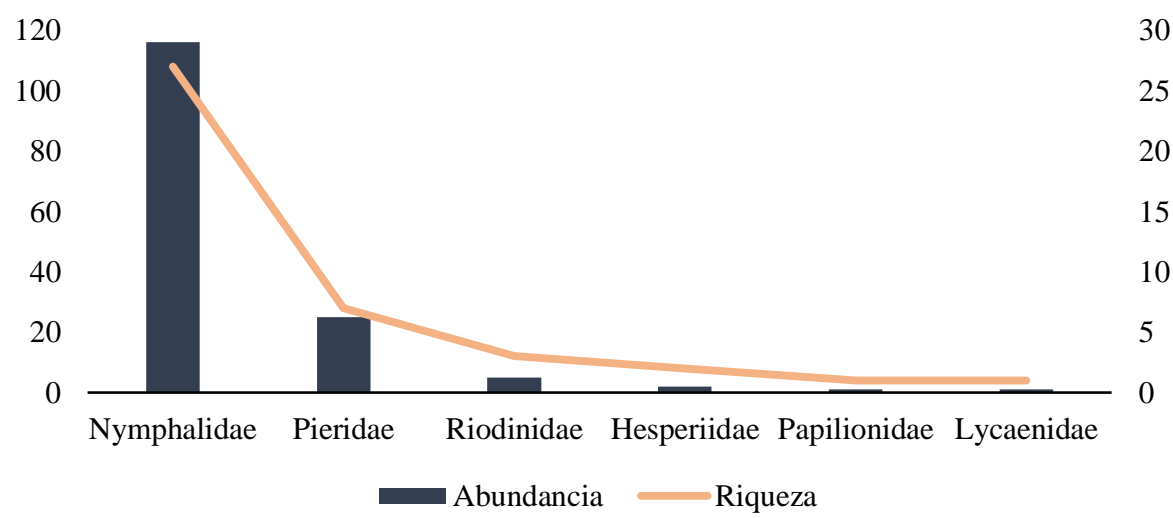

Fig. 5. Abundancia y riqueza de especies por familia de mariposas colectadas en trampas Van Someren-Rydon

La mayor abundancia y riqueza de especies colectadas con las trampas se dio en la estación A, con 53 individuos y 17 especies, respectivamente y la menor abundancia y riqueza de especies se dio en la estación $\mathrm{C}$, en la cual se registraron 19 individuos y ocho especies (Fig. 6). 


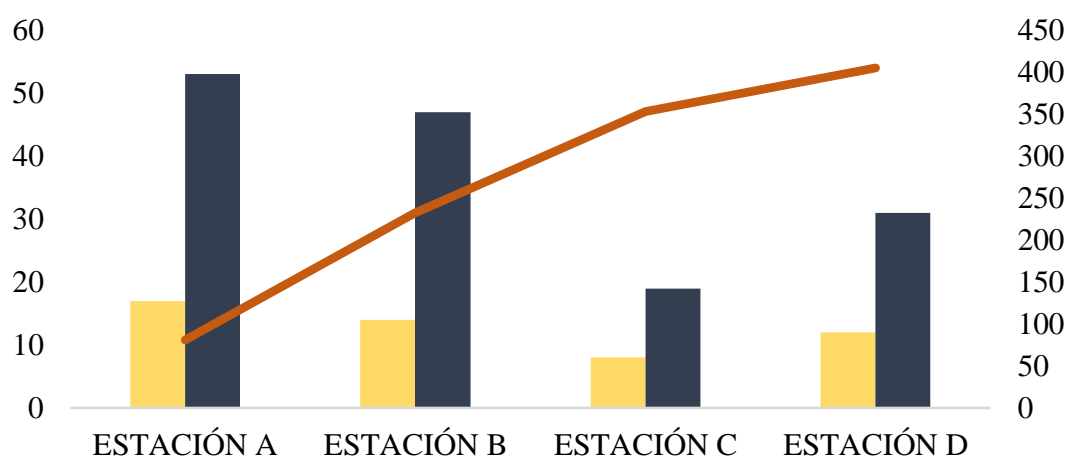

RIQUEZA ABUNDANCIA $\longrightarrow$ ALTITUD (m s.n.m.)

Fig. 6. Abundancia y riqueza de especies colectadas con la trampa VSR en las diferentes estaciones de muestreo

La red entomológica fue el método con mayor efectividad en cuanto a la riqueza y abundancia de especies con un $83,5 \%$ del total de individuos colectados, lo cual superó considerablemente el número de colectas con las trampas cebadas, lo que representa el 16, 5\% del total de colecta.

\section{DISCUSIÓN}

DeVries (1987), reporta 550 especies de mariposas para Panamá, por lo que la riqueza de especies registradas en el trayecto principal hacia la meseta de Chorcha equivale al $20.3 \%$ del total de especies de mariposas del país. Esta riqueza es relativamente alta con respecto a los $4 \mathrm{~km}$ muestreados del área de estudio; además, la meseta de Chorcha es una zona frecuentemente intervenida por el hombre para la ganadería, la agricultura y la ubicación de torres de alta tensión eléctrica.

Santos \& Cambra (2003), reportan 152 especies de mariposas en el Parque Nacional Darién, sin embargo, en este estudio se registraron 112 especies de mariposas. Esto se explica ya que la meseta de Chorcha es una zona perturbada en comparación al Parque Nacional Darién, la cual es el área protegida de mayor tamaño en Panamá, con una extensión de 579,000 hectáreas (Santos, 2003) y no presenta una elevada actividad antropogénica.

Por otra parte, Acosta (2007), registró un total de 3,588 individuos, comparado con los 907 individuos registrados en este estudio, lo cual indica una abundancia relativamente baja; sin embargo, cabe resaltar que dicho autor se centró en mariposas y polillas de hábitos diurnos Tecnociencia, Vol. 23, $N^{\circ} 1$ 
durante un año de muestreo, mientras que el presente estudio solo se enfocó en mariposas durante seis meses de muestreo.

Las especies de mariposas más frecuentes en esta investigación pertenecen a la familia Nymphalidae, a excepción de Heraclides thoas, de la familia Papilionidae. Según DeVries (1987), Nymphalidae es la familia más amplia de mariposas a nivel mundial, quienes se pueden encontrar en la mayoría de los ecosistemas, debido a que es un grupo de mariposas de fácil adaptación a ambientes perturbados.

M. helenor fue la especie más frecuente con 23 registros, esta especie se caracteriza por ser activa durante el día y por volar en senderos abiertos, además está presente durante todo el año (DeVries, 1987). Lo anterior explica la frecuencia de esta mariposa en la meseta de Chorcha, ya que es un área que presenta una gran variedad de ecosistemas en los cuales esta especie se puede desarrollar.

Heraclides thoas se registró en 22 ocasiones, esta mariposa vuela en áreas donde hay abundante incidencia del sol, y se encuentran en diversos ecosistemas como pastizales y áreas boscosas (DeVries, 1997). Esta especie habitualmente visita a una gran variedad de flores durante la época lluviosa o seca (Oñate-Ocaña, 2006), lo que puede explicar su frecuencia en este estudio.

Otras especies de mariposas frecuentes fueron: Heliconius melpomene, Anartia fatima, Heliconius hecale y Pierella luna, todas con 22 registros. Estas especies pertenecen a la familia Nymphalidae, con la mayor distribución a nivel mundial y se encuentra presente en una gran cantidad de hábitats (González-Valdivia et al. 2016).

Estas mariposas son comúnmente vistas alimentándose del néctar de las flores, lo que explica la alta frecuencia de estos ejemplares en la meseta de Chorcha, ya que son especies que se han adaptado a lugares intervenidos por el ser humano (González-Valdivia et al. 2016).

Se registraron 574 individuos en la época lluviosa, representando el $63.3 \%$ del total de mariposas colectadas. Esto contrasta lo presentado por Acosta (2007), donde la mayor cantidad de mariposas registradas fue durante la época seca, por lo que esta elevada abundancia se puede ver favorecida por los altos niveles de precipitación en la meseta de Chorcha, que induce a salir los nuevos brotes foliares y el desarrollo 
floreal de las plantas, condición necesaria para la alimentación y desarrollo larvario de las mariposas (Boom-Urueta et al. 2013).

En la época seca se registraron 333 individuos, lo cual representa el $36.7 \%$ de abundancia total de mariposas, esto se puede explicar por la disminución de la floración de plantas, por lo que algunas especies de mariposas pueden haber volado a otro sitio por su especificidad alimenticia (Marín-Gómez, 2011).

Además, muchas especies de mariposas no se alimentan específicamente de flores, sino también de sales presentes en el suelo, excrementos o frutas en descomposición (DeVries, 1987). Ciertas especies de mariposas pueden estar limitadas a una temporada específica durante el año, lo que justifica la riqueza de especies presentes en ambas épocas de muestreo (Moyers \& Cano, 2009).

En este estudio, los elevados niveles de temperatura y baja precipitación en la época seca, influyeron de manera negativa en la abundancia de mariposas. Boom-Urueta et al. (2013), advierten que las lluvias desempeñan un rol importante en la riqueza y abundancia de las mariposas, ya que los elevados índices de precipitación van relacionados a una humedad relativa alta, lo cual beneficia la actividad de las mariposas, puesto que los valores bajos de humedad relativa producen pérdidas de agua, provocando que las mariposas se deshidraten (Moyers \& Cano, 2009). Además, la presencia de una quebrada en la meseta de Chorcha puede influir en la regulación de las condiciones ambientales y de esta manera tener un impacto en la dinámica poblacional de las mariposas (Boom-Urueta et al. 2013).

En el muestreo realizado con las trampas VSR, se capturaron un total de 150 mariposas pertenecientes a seis familias y 51 especies. De estas, 70 mariposas fueron atraídas al cebo de banano fermentado, mostrando ser el más efectivo para su captura; esto puede estar relacionado a que muchas especies de mariposas tienden a preferir el cebo de fruta fermentada debido a los fluidos azucarados y producción de alcohol dada la descomposición del fruto (Hamer et al. 2006).

El cebo de heces de ganado fue el segundo en cuanto al número de individuos colectados (38); esta carnada atrae a diversas mariposas que se encuentran en la búsqueda de sales minerales como requerimiento para su metabolismo (Vélez \& Salazar, 1991). Por otra parte, 27 mariposas prefirieron el cebo de fruta fresca, el cual le proporciona azúcares y agua a las mariposas. El cebo de pescado descompuesto Tecnociencia, Vol. 23, $N^{\circ} 1$ 
registró 15 mariposas, de hecho, este señuelo resulta ser rico en nitrógeno y potasio, dos elementos esenciales para que algunas especies de mariposas realicen sus procesos metabólicos (Kremen, 1994).

En este estudio, el uso de la red entomológica resultó ser el método más efectivo para la colecta de mariposas con el $83.5 \%$ del total de mariposas colectadas, esto se debe a que muchas especies de mariposas buscan zonas donde los rayos del sol puedan incidir directamente, lo que les permite aumentar su temperatura corporal (Moyers \& Cano, 2009).

Lo anterior concuerda con el estudio realizado por Acosta (2007), el autor explica que las mariposas colectadas con la red entomológica se encontraban en hábitats de sabana y bosque con parches de sol. Lo cual puede entenderse a que muchas especies utilizan estos espacios como corredores o rutas migratorias (Moyers \& Cano, 2009).

Las trampas VSR ubicadas en la estación A fueron las que exhibieron la mayor abundancia (53) y riqueza de especies (17), respectivamente. Esta estación se caracteriza por presentar un bosque de galería a lo largo de la Quebrada del Chorro, lo que puede explicar la gran cantidad de mariposas capturadas en esta zona.

Dingle et al. (2005), menciona que muchas especies de mariposas utilizan estos parches boscosos a orillas de las quebradas y ríos como corredores biológicos o rutas de tránsito.

En este estudio no se realizaron muestreos con red entomológica a lo largo de la quebrada, debido a que no era parte de la zona de muestreo; sin embargo, resulta necesario que se hagan investigaciones sobre la diversidad de mariposas a lo largo del bosque de galería de la quebrada del Chorro, puesto que es posible encontrar numerosas especies y evaluar el impacto de la intervención humana en este parche boscoso.

Se colectaron 47 individuos y 14 especies en la estación B, la cual se caracteriza por ser un área boscosa y poco intervenido por el hombre. La gran cantidad de plantas que se localizan en esta zona puede favorecer la riqueza y abundancia de especies de mariposas, siendo utilizadas como plantas hospederas por estos insectos para depositar sus huevos y desarrollar las larvas (Sánchez, 2004).

En la estación C se registraron los índices más bajos de riqueza (8) y abundancia de mariposas (19). Esta zona se diferencia por presentar laderas pronunciadas con parches boscosos que se encuentran en Araúz G. \& Colaboradores 
transición entre el bosque maduro de la estación B, y los herbazales de la estación D. Es importante destacar que los niveles de abundancia y riqueza de mariposas pueden ser bajos, debido a que esta zona no presenta muchas especies de plantas hospederas para las especies estudiadas, además de que las mariposas pueden estar trasladándose a otras zonas con abundantes fuentes alimenticias permanentes.

En la estación D se registraron 31 individuos pertenecientes a 12 especies esta zona presenta abundantes matorrales que son utilizados para la ganadería, además cuenta con pocos parches boscosos a la orilla de la quebrada del Chorro y a orilla del lago de la meseta.

Las mariposas registradas en esta zona pueden estar acostumbradas a las permanentes fuentes de alimentación como las heces de ganado, restos de pescado en descomposición presentes en el lago. En contraste, el aumento de la superficie de los potreros y deforestación para la ubicación de las torres de alta tensión eléctrica, pueden estar influyendo negativamente en las poblaciones de las mariposas de la meseta de Chorcha.

\section{CONCLUSIONES}

Se registraron 907 mariposas distribuidas en 112 especies. Las familias con mayor número de especies fueron Nymphalidae, Pieridae y Riodinidae, respectivamente.

La época lluviosa presentó los valores más altos de abundancia y riqueza de especies con 554 individuos y 168 especies respectivamente, en la época seca se registraron 353 individuos y 129 especies.

La especie de mariposa más frecuente fue Morpho helenor, la cual se registró durante 23 días de colecta.

La red entomológica fue el método más efectivo para la recolección de mariposas, con 757 individuos colectados. El cebo de fruta descompuesta fue el más efectivo para atraer a las mariposas (70 individuos). 


\section{AGRADECIMIENTOS}

A Víctor Villarreal y Alberto Quiel por la colaboración en la colecta de mariposas. A Jairo Díaz y Adair Quiróz por su apoyo en esta investigación.

\section{REFERENCIAS}

Acosta, L. 2007. Macro lepidópteros de hábitos diurnos en la meseta de Chorcha, Chiriquí, Panamá, 2005-2006. Tesis de licenciatura. Universidad Autónoma de Chiriquí. 155 pp.

Aiello, A. 2001. Las orugas de Panamá., In: Stanley Heckadon-Moreno (ed.), Panamá: Puente Biológico: Las Charlas Smithsonian del Mes: 1996-1999. Instituto Smithsonian de Investigaciones Tropicales, Balboa, Republica de Panamá. Pg. 118-125. xx + 233 pp. ISBN 9962614-01-5].

Aiello, A. 1992. Dry season strategies of two Panamanian butterfly species, Anartia fatima (Nymphalinae) and Pierella luna (Satyrinae) (Lepidoptera: Nymphalidae). In: D. Quintero Arias \& A. Aiello (Ed.), Insects of Panama y Mesoamerica: selected studies: 573-575. Oxford, New York \& Tokyo: Oxford University Press.

Andrade-C, G. 1990. Clave para las familias y subfamilias de Lepidóptera: Rhopalocera de Colombia. Caldasia, 16 (77), 197-200.

Basset, Y., H. Barrios, S. Segar, RB Srygley, A. Aiello, A.D. Warren, F. Delgado, J. Coronado, J. Lezcano, S. Arizala, M. Rivera, F. Pérez, R. Bobadilla, Y. López \& J.A. Ramírez. 2015. "The Butterflies of Barro Colorado Island, Panama: Local Extinction since the 1930s." PloS One 10 (8):1-22. https://doi.org/10.1371/journal.pone.0136623

Boom-Urueta, C., L. Seña-Ramos, M. Vargas-Zapata \& N. MartínezHernández. 2013. Mariposas Hesperioidea y Papilionoidea (Insecta: Lepidoptera) en un Fragmento de Bosque Seco Tropical, Atlántico, Colombia. Boletín Científico Centro de Museos Museo de Historia Natural, 17, 149-167.

Castro, A. 1986. Análisis fisiográfico de la meseta de Chorcha. Tesis de licenciatura. Universidad Autónoma de Chiriquí, Panamá. 208 pp. 
Consejo Municipal de David, 2017. Acuerdo ํ29.

DeVries, P.J. 1987. Butterflies of Costa Rica and their natural history. Papilionidae, Pieridae, Nymphalidae. Princeton University Press. 327 p.

DeVries, P.J. 1997. Butterflies of Costa Rica and their natural history: Volume II (Riodinidae). Princeton University Press. New Jersey 288 p.

Dingle, H., M.P. Zalucki, W.A. Rochester \& T. Armijo-Prewitt. 2005. Distribution of the monarch butterfly Danaus plexippus (L.) (Lepidoptera: Nymphalidae) in western North America. Biol. J. Linn. Soc., 85: 491-500.

Empresa de Transmisión Eléctrica S.A. (ETESA). 2020. Gerencia de Hidrometeorología y Estudios. http://www.hidromet.com.pa/

García-Barros, Enrique; Helena Romo; Víctor Sarto i Monteys; Miguel L. Munguira; Joaquín Baixeras; Antonio Vives Moreno y José Luis Yela García6. 2015. Orden Lepidoptera. Revista IDE@ - SEA, 65, 1-21.

González-Valdivia N., C. Pozo, S. Ochoa-Gaona, B. Ferguson, E. Cambranis, O. Lara, I. Pérez-Hernández, A. Ponce-Mendoza \& C. Kampichler. 2016. Nymphalidae frugívoras (Lepidoptera: Papilionoidea) asociadas a un ecomosaico agropecuario y de bosque tropical lluvioso en un paisaje del sureste de México. Revista mexicana de biodiversidad, $\quad 87(2), \quad 451-464$. https://dx.doi.org/10.1016/j.rmb.2016.04.003

Google Earth (s.f). [Mapa de la meseta de Chorcha. Panamá en Google maps]. Recuperado el 05 de septiembre, 2020, de: https://earth.google.com/web/@0,0,0a,22251752.77375655d,35y,0h,0t , $0 \mathrm{r}$

Hamer, K.C., J.K. Hill, S. Benedick, N. Mustaffa, V.K. Chey \& M. Maryati. 2006. Diversity and Ecology of carrion and fruit-feeding butterflies in Bornean rain forest. Journal of Tropical Ecology, 22: 2535 .

Huntington E.I. 1932. A list of the Rhopalocera of Barro Colorado Island, Canal Zone, Panama. Bull Am Mus Nat Hist 113: 191-230.

Kremen, C. 1994. Monitoreo ecológico: Una necesidad vital para Tecnociencia, Vol. 23, $N^{\circ} 1$ 
integrar programas de conservación y desarrollo en los trópicos. Conservation Biology, 8, 388-397.

Mallet J. 1986. Hybrid Zones of Heliconius Butterflies in Panama and the Stability and Movement of Warning Colour Clines. Heredity 56: 191-202

Marín-Gómez, Oscar Humberto; Rocío García C.; Walter Fabio Gómez M. y Wilson Pinzón L. 2011. Diversidad de Mariposas y su Relación con la Fenología Reproductiva de Inga Ornata Kunth (Mimosoidae) en un agroecosistema ganadero del Quindío, Colombia. Boletín Científico Centro de Museos, Museo de Historia Natural., 15 (2), 105-118.

Moyers y Cano. 2009. Fenología de la comunidad de mariposas diurnas y su relación con la fenología floral de las plantas y otros factores ambientales, pp. 411-419 In A. Lot y Z. Cano-Santana [eds.], Biodiversidad del Pedregal de San Ángel. UNAM, Reserva Ecológica del Pedregal de San Ángel y Coordinación de la Investigación Científica, D.F. México.

Ollerton J. 1999. La evolución de las relaciones polinizador - planta en los artrópodos. Boletín Sociedad Entomológica Aragonesa. 26: 741758.

Oñate, L.O., M.O. Trujano, J.B. Llorente, A.M. Luis y I.F. Vargas. 2006. Patrones de distribución de la familia Papilionidae (Lepidoptera). In Componentes bióticos principales de la entomofauna mexicana, vol. II, J. J. Morrone y J. B. Llorente (eds.). Las Prensas de Ciencias, Facultad de Ciencias, UNAM, México, D. F. p. 661-714.

Padilla A.C., A. Thurman, J. MacDonald y YJ Añino. 2020. Lista sinóptica y frecuencia de recolecta de mariposas (Lepidoptera: Rhopalocera) en Playa Corona, San Carlos, Panamá. Poeyana, 510: 8992.

Rodríguez, C. 1996. El género Morpho en Colombia: II. Clave taxonómica para las especies presentes en Colombia. Agronomía Colombiana, 13 (1), 1-22.

Sánchez, R. 2004. Protocolo de cría para dos especies de mariposas, Ascia monuste y Leptophobia aripa (Lepidoptera: Pieridae) bajo Araúz G. \& Colaboradores 
condiciones controladas en el municipio de la Mesa, Cundinamarca (Tesis de pregrado). Pontificia Universidad Javeriana. Bogotá.

Santos, A. 2012. Mariposas Papilionoidea y Hesperioidea (Insecta: Lepidoptera) de la Isla Majé, Comarca Guna de Madungandí, Panamá. Tecnociencia, 14, 32-54.

Santos A. y R. Cambra. 2003. Mariposas del Parque Nacional Darién: Nymphalidae, Papilionidae y Pieridae (Lepidoptera) depositadas en el Museo de Invertebrados G. B. Fairchild, Universidad de Panama. Tecnociencia 5(2): 23-33.

Silberglied R., A. Aiello, y D.M. Windsor. 1980. Disruptive coloration in butterflies: Lack of support in Anartia fatima. Science. 209 (4456): 617-619.

Urretabizkaya, N. 2010. Insectos Perjudicales de Importancia Agronómica I. Lepidópteros. Buenos Aires: INTA.

Vélez, J. y J. Salazar. 1991. Mariposas de Colombia: 167 pp. Villegas ed. Bogotá. Http: //home.att.net/-bret69 (R. Aronheim - Papilios of the World web page.

Villareal, H., M. Álvarez, S. Córdoba, F. Escobar, G. Fagua, F. Gast, H. Mendoza, M. Ospina, y A.M. Umaña. 2004. Manual de métodos para el desarrollo de inventarios de biodiversidad. Programa de Inventarios de Biodiversidad. Instituto de Investigación de Recursos Biológicos Alexander von Humboldt. Bogotá, Colombia. 236 pp.

Warren, A. D., K. J. Davis, E. M. Stangeland, J. P. Pelham, K. R. Willmott y N. V. Grishin. 2016. Illustrated Lists of American Butterflies. [21-XI-2017] http://www.butterfliesofamerica.com 


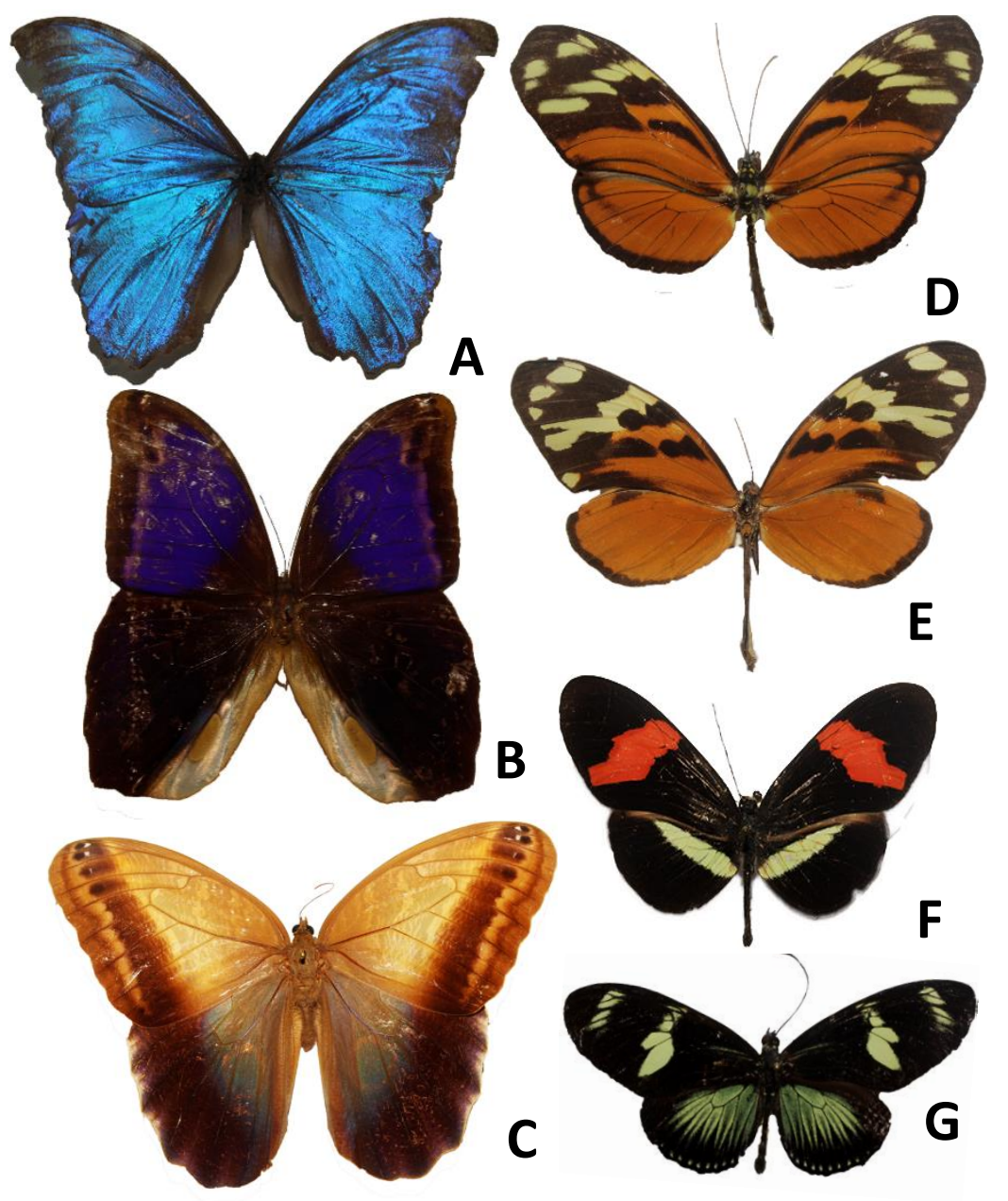

Fig. 7. A - G Nymphalidae: A. Morpho menelaus. B. Eryphanis lycomedon. C. Caligo telamonius. D. Heliconius ismenius. E. Melinaea scylax. F. Heliconius erato petiverana. G. Heliconius doris. 


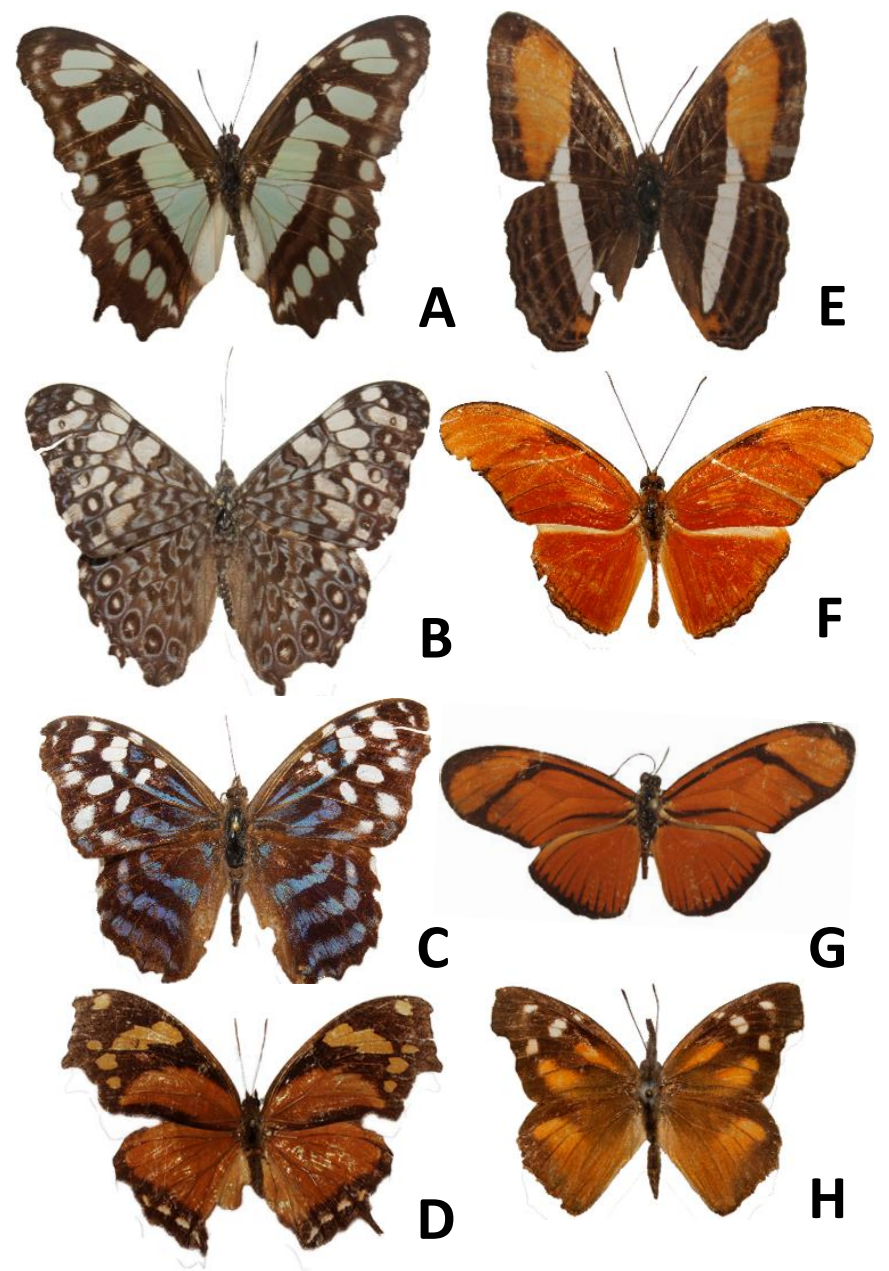

Fig. 8. A - H Nymphalidae: A. Siproeta stelenes. B. Hamadryas feronia. C. Myscelia leucocyana. D. Consul fabius. E. Adelpha cytherea. F. Dryas iulia. G. Eueides aliphera. H. Libytheana carinenta. 


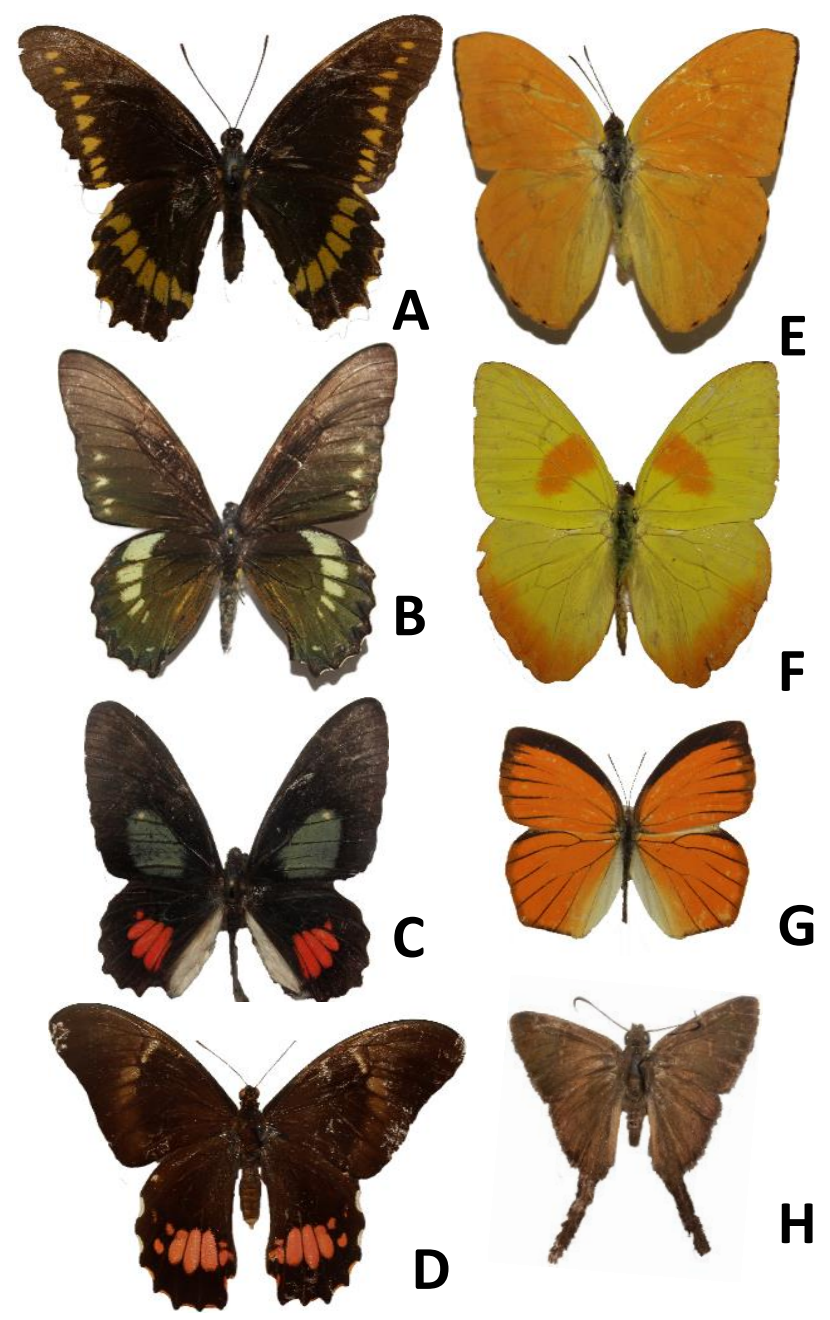

Fig. 9. A - D Papilionidae: A: Battus polydamas. B: Battus ingennus. C: Parides panares. D. Heraclides anchisiades. E - G Pieridae. E. Phoebis argante. F. Phoebis philea. G. Pyrisitia proterpia. H. Hesperiidae: Urbanus simplicius. 